\title{
A study to assess the expertise and training in percutaneous endoscopic gastrostomy (PEG) insertion among trainee gastroenterologists
}

\author{
J. Saunders, B. Colleypriest and H. Gordon \\ Gastroenterology Department, Royal Hampshire County Hospital, Romsey Road, Winchester SO22 5DG, UK
}

PEG insertion is a procedure performed in most endoscopy units nationwide. It has considerable benefits but carries a significant mortality rate. A direct procedure-related rate between $0.7 \%$ and $2.0 \%$ is commonly quoted. The $30 \mathrm{~d}$ mortality varies from $10 \%$ to $24 \%$ in published series. The 2004 National Confidential Enquiry into Patient Outcome and Deaths audit on endoscopy has highlighted patient selection and the timing of the procedure as the important factors in reducing mortality rates ${ }^{(1)}$. It recommends that all patients are reviewed by a multidisciplinary team before undergoing the procedure. The 2005 Joint Committee on Higher Medical Training gastroenterology curriculum indicates that a minimum number of assessed procedures should be completed before being considered as an independent operator ${ }^{(2)}$.

The present study aims to determine the level of practical training and experience of PEG insertion among gastroenterology trainees. It also aims to determine the extent of participation within specialist nutrition teams. All gastroenterology specialist registrars (SpR) in the Wessex deanery received an anonymised questionnaire.

In total $73 \%$ of the thirty SpR responded, of whom $95 \%$ described themselves as independent upper gastrointestinal endoscopists. A total of $82 \%$ had received no formal training in PEG insertion techniques, such as a Joint Advisory Group approved course. Only one individual had completed ten directly-observed procedure assessments for PEG insertion. Two trainees reported that they had been supervised in fewer than ten procedures before performing PEG insertions independently. Only one SpR worked in a unit where PEG were performed on a separate endoscopy list. A total of $77 \%$ of trainees worked in hospitals that had specialist nutrition teams but only $32 \%$ had dedicated time with the team as part of their timetable. Only $14 \%$ of trainees reported that patients were not seen on the ward before the procedure, they equally had no nutrition team in their hospitals. Five SpR worked in hospitals where the endoscopist consents the patient. In circumstances where the patient was unable to consent, three registrars reported that the endoscopist rather than the consultant in charge of the patient's care completed the consent form.

PEG insertion has the highest mortality rate of any procedure that most trainees will undertake. In order to improve the mortality rate, trainees should have better access to practical endoscopy courses and need to ensure that documentation of competence is completed. Appropriate patient selection is probably the most important component in reducing both procedure-related and $30 \mathrm{~d}$ mortality and most authorities agree that it is best tackled by a multidisciplinary team. The current study suggests that specialist nutrition team involvement in PEG insertion and SpR training is inadequate and that trainees would benefit from having more time dedicated to nutrition training.

1. Cullinane M, Gray AJG, Hargraves CMK et al. (2004) Scoping our Practice. The 2004 Report of the National Confidential Enquiry into Patient Outcome and Death. London: NCEPOD.

2. Joint Committee on Higher Medical Training (2005) Higher Medical Training Curriculum for Gastroenterology 2005. London: Royal College of Physicians. 